PROCEEDINGS OF THE

AMERICAN MATHEMATICAL SOCIETY

Volume 128, Number 5, Pages 1507-1513

S 0002-9939(00)05109-1

Article electronically published on February 7, 2000

\title{
COMPACTIFYING SUFFICIENTLY REGULAR COVERING SPACES OF COMPACT 3-MANIFOLDS
}

\author{
ROBERT MYERS
}

(Communicated by Ronald A. Fintushel)

\begin{abstract}
In this paper it is proven that if the group of covering translations of the covering space of a compact, connected, $\mathbf{P}^{2}$-irreducible 3 -manifold corresponding to a non-trivial, finitely-generated subgroup of its fundamental group is infinite, then either the covering space is almost compact or the subgroup is infinite cyclic and has normalizer a non-finitely-generated subgroup of the rational numbers. In the first case additional information is obtained which is then used to relate Thurston's hyperbolization and virtual bundle conjectures to some algebraic conjectures about certain 3-manifold groups.
\end{abstract}

\section{INTRODUCTION}

A non-compact 3-manifold $V$ is almost compact if it is homeomorphic to a compact 3-manifold $W$ minus a closed subset of its boundary; if $g: V \rightarrow W$ is the corresponding embedding, then the pair $(W, g)$ is called a manifold compactification of $V$; this term is also applied to $W$ itself. Simon has conjectured [29] that if $M$ is a compact, connected, $\mathbf{P}^{2}$-irreducible 3 -manifold (possibly with non-empty boundary) and $H$ is a finitely-generated subgroup of $\pi_{1}(M)$, then the covering space $\widetilde{M}$ of $M$ corresponding to $H$ is almost compact. The case in which $H$ is trivial has received considerable attention. (See, for example, [34, [12], [10], 31], [1] and 20].) For $H$ non-trivial the conjecture is known to hold if $M$ is Haken and $H$ is abelian or peripheral [29, [16, if $M$ is hyperbolic and $H$ is indecomposable with respect to free products [2], if $H$ is $\mathbf{Z} \oplus \mathbf{Z}$ [12], if $M$ is laminar and $H$ is $\mathbf{Z}[8$, and if $\pi_{1}(M)$ is automatic and $H$ is regular [19].

The situation in which $H$ is a non-trivial normal subgroup of $\pi_{1}(M)$, i.e. in which $p: \widetilde{M} \rightarrow M$ is a non-universal regular covering, is well understood. (See [30], [14, 13.) If $\widetilde{M}$ is non-compact, then $M$ must either be a bundle over $S^{1}$ with fiber a compact surface $F$, a union of two copies of a twisted $I$-bundle over a compact surface $F$ along the associated $\partial I$-bundles, or a Seifert fibered space. In the first two cases $H$ is conjugate to a subgroup of $\pi_{1}(F)$. In the third case it is conjugate to a subgroup of the infinite cyclic group generated by a regular fiber. It then follows easily that $\widetilde{M}$ is almost compact.

Received by the editors October 7, 1997 and, in revised form, June 1, 1998.

1991 Mathematics Subject Classification. Primary 57M10; Secondary 57N10, 57M60.

Key words and phrases. 3-manifold, covering space, compactification, hyperbolic 3-manifold. Research at MSRI is supported in part by NSF grant DMS-9022140. 
In making his conjecture Simon expressed the hope "that the regularities inherent even in irregular covering spaces" would ensure that $\widetilde{M}$ is almost compact. This paper considers one obvious sort of regularity which sometimes occurs, namely the condition that $H$ has infinite index in its normalizer $N(H)=\left\{g \in \pi_{1}(M) \mid g H^{-1}=\right.$ $H\}$, i.e. in which the group of covering translations $A u t(p) \cong N(H) / H$ is infinite.

Theorem 1. Let $M$ be a compact, connected, $\mathbf{P}^{2}$-irreducible 3-manifold. Let $H$ be a non-trivial, finitely-generated subgroup of $\pi_{1}(M)$. Let $p: \widetilde{M} \rightarrow M$ be the covering map such that $p_{*}\left(\pi_{1}(\widetilde{M})\right)=H$. Suppose the group of covering translations is infinite. Then one of the following holds:

(1) $\widetilde{M}$ has manifold compactification $(F \times[0,1], g)$, where $F$ is a compact, connected surface (possibly with non-empty boundary); hence $H \cong \pi_{1}(F)$. In addition one has the following commutative diagram.

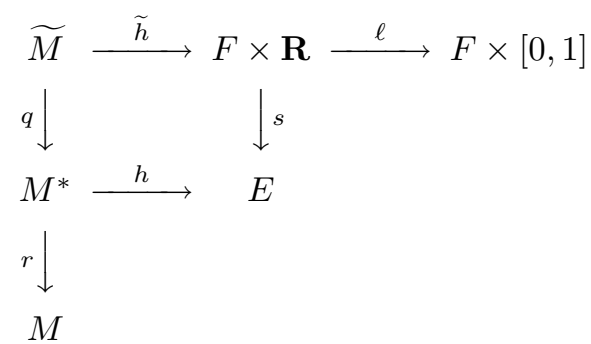

In this diagram $r \circ q=p, \ell \circ \widetilde{h}=g$, $\ell$ is the standard embedding with image $F \times(0,1), q$ and $s$ are infinite cyclic covering maps, $(E, h)$ is a manifold compactification of $M^{*}, E$ is a bundle over $S^{1}$ with fiber $F$, and $h_{*}\left(q_{*}\left(\pi_{1}(\widetilde{M})\right)\right)=\pi_{1}(F)=s_{*}\left(\pi_{1}(F \times \mathbf{R})\right)$. Moreover either

(a) $M^{*}$ is compact, $r$ is finite sheeted, and $h$ and $\widetilde{h}$ are homeomorphisms (hence $M$ is finitely covered by a surface bundle over $S^{1}$ ), or

(b) $M^{*}$ is non-compact, $r$ is infinite sheeted, $r_{*}\left(\pi_{1}\left(M^{*}\right)\right)$ has a $\mathbf{Z} \oplus \mathbf{Z}$ subgroup $A$, and $H$ is a free group. Moreover either

(i) $A$ is not conjugate to a subgroup of the fundamental group of an incompressible component of $\partial M$, or

(ii) $A$ is conjugate to such a subgroup, and $H$ is an infinite cyclic subgroup of $A$.

(2) $H$ is infinite cyclic, $N(H)$ is a non-finitely-generated subgroup of the additive group of rational numbers, and the group of covering translations is a nonfinitely-generated infinite torsion group.

The proof of this theorem occupies sections 2 and 3 . In section 2 it is shown that if the group of covering translations has an element of infinite order, then case (1) holds. In section 3 it is shown that if this group does not have an element of infinite order, then case (2) holds.

Note that if $M$ is orientable and $\pi_{1}(M)$ contains a $\mathbf{Z} \oplus \mathbf{Z}$ subgroup, then either $M$ contains an incompressible torus or $M$ is Seifert fibered [34, [1], [27], 18], [3], 6]. If $M$ is non-orientable and $\pi_{1}(M)$ contains a $\mathbf{Z} \oplus \mathbf{Z}$ subgroup, then since $M$ is Haken [13] it must contain an incompressible torus or Klein bottle [27], [16].

Note also that if $M$ is orientable and satisfies the conclusion of Thurston's geometrization conjecture [32], i.e. $M$ is Haken, hyperbolic, or Seifert fibered, then case (2) cannot occur since Haken manifold groups [28] and hyperbolic groups [23] 
cannot have infinitely divisible elements and Seifert fibered spaces with infinite fundamental groups are finitely covered by Haken manifolds [5]. If $M$ is non-orientable, then it is Haken, and so again case (2) cannot occur. These observations immediately imply the following result.

Corollary 1. Let $M$ be a compact, connected, $\mathbf{P}^{2}$-irreducible 3-manifold. Suppose $\partial M$ has a component with negative Euler characteristic and for each $\mathbf{Z} \oplus \mathbf{Z}$ subgroup $A$ of $\pi_{1}(M)$ there is an incompressible component $T$ of $\partial M$ such that $A$ is conjugate to a subgroup of $\pi_{1}(T)$. Then each non-trivial, finitely-generated subgroup of $\pi_{1}(M)$ either has finite index in its normalizer or is an infinite cyclic subgroup of such an $A$.

As another application of this theorem we give a pair of conjectures about certain 3-manifold groups which, taken together, are equivalent to the hyperbolic case of the geometrization conjecture, taken together with the conjecture that closed hyperbolic 3-manifolds are finitely covered by surface bundles over $S^{1}$. We also give an analogous conjecture which is equivalent to the bounded case of this "virtual bundle conjecture." This material is contained in section 4 .

We remark that several of the compactification results mentioned earlier can be given short, elegant proofs using the Tucker compactification theorem [33], which states that a connected, $\mathbf{P}^{2}$-irreducible, non-compact 3 -manifold is almost compact if and only if each component of the complement of each compact polyhedron in the manifold has finitely generated fundamental group. In [19] Mihalik generalizes this criterion to the group theoretic notion of a tame pair $(G, H)$, where $G$ is a finitely presented group and $H$ is a finitely generated subgroup of $G$. In our context it turns out that the pair $\left(\pi_{1}(M), H\right)$ is tame if and only if $\widetilde{M}$ is almost compact. It seems unlikely that the element of infinite order case of Theorem 1 generalizes as stated to the context of tame pairs, but it might be interesting to find conditions under which a generalization is possible.

\section{The ElEMENT OF INFINITE ORDER CASE}

Lemma 1. If Aut ( $p)$ has an element $\varphi$ of infinite order, then case (1) holds.

Proof. Let $M^{*}=\widetilde{M} /\langle\varphi\rangle$. Then $p$ factors as $\widetilde{M} \stackrel{q}{\rightarrow} M^{*} \stackrel{r}{\rightarrow} M$. By [17] and [4] $M^{*}$ is $\mathbf{P}^{2}$-irreducible. $\pi_{1}\left(M^{*}\right)$ has a finitely-generated normal subgroup $q_{*}\left(\pi_{1}(\widetilde{M})\right) \cong H$ with infinite cyclic quotient. If $M^{*}$ is compact, then by the Stallings fibration theorem [30] it is a bundle over $S^{1}$ with fiber a compact surface $F$ such that $\pi_{1}(F)=$ $q_{*}\left(\pi_{1}(\widetilde{M})\right)$. Thus $(1)$ (a) follows. So assume that $M^{*}$ is not compact.

Let $C$ be the Scott compact core of $M^{*}$ [25, i.e. $C$ is a compact, connected, 3dimensional submanifold of int $M^{*}$ such that $\pi_{1}(C) \rightarrow \pi_{1}\left(M^{*}\right)$ is an isomorphism. Since $M^{*}$ is irreducible we may assume by, if necessary, adjoining 3-balls in $M^{*}-$ $\operatorname{int} C$ along 2-sphere components of $\partial C$, that $C$ is irreducible. Then as above $C$ is a surface bundle over $S^{1}$. In particular $\partial C$ consists of tori and Klein bottles. If some component $S$ of $\partial C$ is compressible in $C$, then the 2 -sphere obtained by compressing $S$ bounds a 3-ball in $C$, and hence $C$ is a solid torus or solid Klein bottle, and so $\pi_{1}\left(M^{*}\right) \cong \mathbf{Z}$. But this implies that $H$ is trivial, a contradiction. Thus $\partial C$ is incompressible in $C$ and hence in $M^{*}$.

Fix a component $S$ of $\partial C$. Let $X$ be the component of $M^{*}-\operatorname{int} C$ which meets $S$. Then $S \rightarrow X$ is a homotopy equivalence. If $X$ is compact, then it is homeomorphic to $S \times[0,1]$, where $S \times\{0\}=S$ and $S \times\{1\}$ is a component of $\partial M^{*}$ [34], [13]. 
Suppose now that $X$ is non-compact.

Assume that $S$ is a torus. Let $f: \widehat{M} \rightarrow M$ be the covering map such that $f_{*}\left(\pi_{1}(\widehat{M})\right)=r_{*}\left(\pi_{1}(S)\right)$. If $M$ is closed, then by [12] $\widehat{M}$ is homeomorphic to $S^{1} \times$ $S^{1} \times \mathbf{R}$. If $\partial M \neq \emptyset$, then $M$ is Haken, and so by [29] or [16] one has that $\widehat{M}$ is almost compact. Thus in each of these cases $\widehat{M}$ has manifold compactification $S^{1} \times S^{1} \times[0,1]$. Now $f$ factors as $\widehat{M} \stackrel{u}{\rightarrow} M^{*} \stackrel{r}{\rightarrow} M$. The embedding of $X$ in $M^{*}$ lifts to a copy $\widehat{X}$ of $X$ in $\widehat{M}$. By Corollary 3.2 of 34 the lifting $\widehat{S}$ of $S$ is isotopic to $S^{1} \times S^{1} \times\{0\}$. Thus $\widehat{X}$ has manifold compactification, say, $S^{1} \times S^{1} \times[0,1]$. Hence the same is true of $X$.

Now assume that $S$ is a Klein bottle. We let $k: M^{+} \rightarrow M^{*}$ be the orientable double cover of $M^{*}$. Then $(X, S)$ is double covered by the pair $\left(X^{+}, S^{+}\right)$, where $S^{+}$ is a torus and $S^{+} \rightarrow X^{+}$is a homotopy equivalence. We apply the argument of the previous paragraph to conclude that $X^{+}$has manifold compactification $S^{1} \times S^{1} \times$ $[0,1]$. We can then see that $X$ is almost compact as follows. Let $K$ be a compact polyhedron in $X$, and let $U$ be a component of $X-K$. Choose a component $U^{+}$ of $k^{-1}(U)$. Then $U^{+}$is a component of $X^{+}-k^{-1}(K)$. Since $k^{-1}(K)$ is a compact polyhedron in the almost compact manifold $X^{+}$we have that $\pi_{1}\left(U^{+}\right)$is finitely generated. Since $\left(\left.k\right|_{U^{+}}\right)_{*}\left(\pi_{1}\left(U^{+}\right)\right)$has finite index in $\pi_{1}(U)$, we have that $\pi_{1}(U)$ is finitely generated. Thus by the Tucker compactification theorem [33] $X$ is almost compact.

We have shown that each component $X$ of $M^{*}-\operatorname{int} C$ has manifold compactification homeomorphic to $S \times[0,1]$, where $S=X \cap C$. Thus $M^{*}$ has manifold compactification $E$ homeomorphic to the surface bundle $C$ with fiber $F$. This establishes the existence of the maps $h, \widetilde{h}, s$, and $g$ in the statement of the theorem.

Since $M^{*}$ is non-compact, some component $S$ of $\partial C$ must cut off a non-compact component $X$ of $M^{*}-\operatorname{int} C$. Suppose $S$ is a torus. Let $A=r_{*}\left(\pi_{1}(S)\right)$. Suppose $A$ is conjugate in $\pi_{1}(M)$ to a subgroup of $\pi_{1}(T)$ for some incompressible component of $\partial M$. It follows that $\pi_{1}(S)$ is conjugate in $\pi_{1}\left(M^{*}\right)$ to a subgroup of $\pi_{1}\left(T^{*}\right)$ for some component $T^{*}$ of $r^{-1}(T)$. By Lemma 5.1 of [34 there is an embedding of $S \times[0,1]$ in $M^{*}$ with $S \times\{0\}=S$ and $S \times\{1\}=T^{*}$. Since $X$ is non-compact, $S \times[0,1]$ contains $C$. Thus $A=\pi_{1}\left(M^{*}\right)$ and we are done. If $S$ is a Klein bottle, we take the orientable double cover $k:\left(M^{+}, S^{+}\right) \rightarrow\left(M^{*}, S\right)$ as above, let $A=(r \circ k)_{*}\left(\pi_{1}\left(S^{+}\right)\right)$, and apply this argument to get a product $I$ bundle in $M^{+}$joining $S^{+}$to a component $T^{+}$of $(r \circ k)^{-1}(T)$. By Proposition 4 of [15] this covers a product $I$ bundle in $M^{*}$ joining $S$ to a component $T^{*}$ of $r^{-1}(T)$. Again this $I$ bundle contains $C$. Any normal subgroup of the Klein bottle group which has infinite cyclic quotient must be an infinite cyclic subgroup of the orientation preserving subgroup, and thus we are done.

Thus case $(1)(\mathrm{b})$ holds.

\section{THE TORSION GROUP CASE}

Lemma 2. If $\operatorname{Aut}(p)$ is a finitely generated infinite group, then it has an element of infinite order.

Proof. Suppose $A u t(p)$ is a torsion group $\Gamma . N(H)$ is finitely generated, and so by 24] is isomorphic to $\pi_{1}(R)$ for some compact 3-manifold $R$. We thus have the exact sequence

$$
1 \rightarrow H \rightarrow \pi_{1}(R) \rightarrow \Gamma \rightarrow 1
$$


By Lemma 2.2 of 14] (see also Lemma 11.9 of [13]) we must have $H \cong \mathbf{Z}$.

If $R$ is orientable, then it must be a Seifert fibered space (see e.g. [13]) and $H$ must be conjugate to a subgroup of the subgroup generated by a regular fiber. It follows that there is an exact sequence

$$
1 \rightarrow K \rightarrow \Gamma \rightarrow G \rightarrow 1
$$

where $K$ is a finite cyclic group and $G$ is an infinite Fuchsian group. $G$ must have an element of infinite order (see e.g. Theorems 12.1 and 12.2 of [13), and thus so does $\Gamma$, contradicting our assumption.

If $R$ is non-orientable, let $k: R^{+} \rightarrow R$ be the orientable double covering. Let $H^{+}=H \cap k_{*}\left(\pi_{1}\left(R^{+}\right)\right)$. Then $H^{+}$has index at most two in $H$ and so is infinite cyclic. Thus $\pi_{1}\left(R^{+}\right)$has an infinite cyclic normal subgroup with quotient group $\Gamma^{+}$a subgroup of index at most two in $\Gamma$. The previous argument shows that $\Gamma^{+}$ has an element of infinite order, and we are done.

Lemma 3. If Aut $(p)$ does not have an element of infinite order, then case (2) holds.

Proof. By Lemma 2 we may assume that $A u t(p)$ is not finitely generated, hence neither is $N(H)$. By Theorem 3.2 of [26] $H$ must be infinite cyclic. Moreover by [5] $N(H)$ must be isomorphic to a subgroup of $\overline{\mathbf{Q}}$, where $\overline{\mathbf{Q}}$ is the unique non-trivial split extension of the additive group $\mathbf{Q}$ of rational numbers by $\mathbf{Z}_{\mathbf{2}}$. Since $M$ is $\mathbf{P}^{2}$-irreducible, $\pi_{1}(M)$ is torsion-free [4, 13], and thus $N(H)$ must lie in $\mathbf{Q}$. Thus case (2) holds.

\section{Some equivalent Sets of COnjectures}

In the following conjectures $M$ denotes a closed, connected, $\mathbf{P}^{2}$-irreducible 3manifold with infinite fundamental group. The first two of these conjectures appeared in [32].

Conjecture 1 (Hyperbolization Conjecture). If $\pi_{1}(M)$ contains no $\mathbf{Z} \oplus \mathbf{Z}$ subgroup, then $M$ is hyperbolic.

Conjecture 2 (Closed Virtual Bundle Conjecture). If $M$ is hyperbolic, then there is some finite sheeted covering space of $M$ which is a surface bundle over $S^{1}$.

Conjecture 3 (Closed Normalizer Conjecture). $\pi_{1}(M)$ has a non-trivial, finitely generated subgroup which has infinite index in its normalizer.

Conjecture 4 (Q Conjecture). Every subgroup of $\pi_{1}(M)$ which embeds in $\mathbf{Q}$ is cyclic.

Corollary 2. Conjectures 1 and 2, taken together, are equivalent to Conjectures 3 and 4, taken together.

Proof. First note that if $M$ is non-orientable, then $M$ is hyperbolic, $M$ is finitely covered by a surface bundle over $S^{1}, \pi_{1}(M)$ has a $\mathbf{Z} \oplus \mathbf{Z}$ subgroup, or $\pi_{1}(M)$ contains a non-cyclic subgroup of $\mathbf{Q}$, respectively, if and only if the corresponding statement is true for its orientable double covering space. (In the hyperbolic case apply Mostow rigidity [21], the Waldhausen-Heil theorem [34, [15], and the fact that $M$ is Haken.) Hence, we may assume that our manifolds are orientable.

Suppose Conjectures 1 and 2 are true. If $\pi_{1}(M)$ has no $\mathbf{Z} \oplus \mathbf{Z}$ subgroup, then clearly Conjectures 3 and 4 hold for $M$. If $\pi_{1}(M)$ has a $\mathbf{Z} \oplus \mathbf{Z}$ subgroup, then 
clearly Conjecture 3 holds for $M$. Moreover as pointed out in the introduction, $M$ either is Seifert fibered or contains an incompressible torus and hence Conjecture 4 holds for $M$.

Now suppose that Conjectures 3 and 4 are true. If $\pi_{1}(M)$ has no $\mathbf{Z} \oplus \mathbf{Z}$ subgroup, then by Theorem 1 we have that $M$ is finitely covered by a surface bundle $M^{*}$ over $S^{1}$. Since $\pi_{1}\left(M^{*}\right)$ also has no $\mathbf{Z} \oplus \mathbf{Z}$ subgroup, the fibered case of Thurston's hyperbolization theorem [32] (see also 22]) implies that $M^{*}$ is hyperbolic. As has been pointed out by Thurston [32] and Gabai [7] the Mostow rigidity theorem [21] implies that $M$ is homotopy equivalent to a hyperbolic 3-manifold. Hence by the topological rigidity of hyperbolic 3-manifolds [9] one has that $M$ is hyperbolic. Thus Conjecture 1 holds for $M$. Now if we start with a hyperbolic 3-manifold $M$, then $\pi_{1}(M)$ has no $\mathbf{Z} \oplus \mathbf{Z}$ subgroup 23 , and so Theorem 1 again implies that $M$ is finitely covered by a bundle. Thus Conjecture 2 holds for $M$.

We now let $N$ denote a compact, connected, $\mathbf{P}^{2}$-irreducible 3 -manifold such that $\partial N$ is a non-empty collection of incompressible tori and Klein bottles. We further assume that each $\mathbf{Z} \oplus \mathbf{Z}$ subgroup of $\pi_{1}(N)$ is peripheral and that $N$ is not an $I$ bundle over a torus or Klein bottle. Note that by Thurston's hyperbolization theorem $32 N$ satisfies this second set of conditions if and only if its interior admits a complete hyperbolic metric of finite volume.

Conjecture 5 (Bounded Virtual Bundle Conjecture). There is some finite sheeted covering space of $N$ which is a surface bundle over $S^{1}$.

Conjecture 6 (Bounded Normalizer Conjecture). $\pi_{1}(N)$ has a non-cyclic, finitely-generated subgroup which has infinite index in its normalizer.

Corollary 3. Conjectures 5 and 6 are equivalent.

Proof. Suppose Conjecture 5 is true. Let $H$ be the image in $\pi_{1}(N)$ of the fundamental group of the fiber. If $H$ were cyclic, then the fiber would be a disk, annulus, or Möbius band, and $N$ would be a solid torus, solid Klein bottle, or an $I$ bundle over a torus or Klein bottle.

Suppose Conjecture 6 is true. Since $N$ is Haken, case (2) of Theorem 1 cannot occur. Since the subgroup is not cyclic, neither can case (1)(b).

\section{REFERENCES}

[1] M. Bestvina and G. Mess, The boundary of negatively curved groups, J. Amer. Math. Soc. 4 (1991), no. 3, 469-481. MR 93j:20076

[2] F. Bonahon, Bouts des variétés hyperboliques de dimension 3 Ann. of Math. (2) 124 (1986), no. 1, 71-158. MR 88c:57013

[3] A. Casson and D. Jungreis, Convergence groups and Seifert fibered 3-manifolds, Invent. Math. 118 (1994), 441-456. MR 96f:57011

[4] D. B. A. Epstein, Projective planes in 3-manifolds, Proc. London Math. Soc. (3) 111961 469-484. MR 27:2968

[5] B. Evans and W. Jaco, Varieties of groups and three-manifolds, Topology 12 (1973), 83-97. MR 48:1207

[6] D. Gabai, Convergence groups are Fuchsian groups, Annals of Math. 136 (1992), 447-510. MR 93m:20065

[7] D. Gabai, Homotopy hyperbolic 3-manifolds are virtually hyperbolic, J. Amer. Math. Soc. 7 (1994), 193-198. MR 94b:57016

[8] D. Gabai and W. Kazez, 3-manifolds with essential laminations are covered by solid tori, J. London Math. Soc. (2) 47 (1993), no. 3, 557-576. MR 94c:57028 
[9] D. Gabai, R. Meyerhoff, and N. Thurston, Homotopy hyperbolic 3-manifolds are hyperbolic, MSRI Preprint Series \#1996-058.

[10] D. Gabai and U. Oertel, Essential laminations in 3-manifolds, Ann. Math. 130 (1989), 41-73. MR 90h:57012

[11] C. Gordon and W. Heil, Cyclic normal subgroups of fundamental groups of 3-manifolds, Topology 14 (1975), no. 4, 305-309. MR 53:4067

[12] J. Hass, H. Rubinstein, and P. Scott, Compactifying coverings of closed 3-manifolds, J. Differential Geometry 30 (1989), 817-832. MR 91d:57009

[13] J. Hempel, 3-Manifolds, Ann. of Math. Studies, No. 86, Princeton, (1976). MR 54:3702

[14] J. Hempel and W. Jaco, Fundamental groups of 3-manifolds which are extensions, Ann. of Math. (2) 95 (1972), 86-98. MR 44:4754

[15] W. Heil, On $P^{2}$-irreducible 3-manifolds, Bull. Amer. Math. Soc. 75, (1969), 772-775. MR 40:4958

[16] W. Jaco, Lectures on three-manifold topology, CBMS Regional Conference Series in Math., No. 43, Amer. Math. Soc. (1980). MR 81k:57009

[17] W. Meeks, L. Simon, S. T. Yau, Embedded minimal surfaces, exotic spheres, and manifolds with postive Ricci curvature, Annals of Math, 116 (1982), 621-659. MR 84f:53053

[18] G. Mess, Centers of 3-manifold groups and groups which are coarse quasi-isometric to planes, preprint.

[19] M. Mihalik, Compactifying coverings of 3-manifolds, Comment. Math. Helv. 71 (1996), no. 3, 362-372. MR 97k:57020

[20] M. Mihalik and S. Tschantz, Tame combings of groups, Trans. Amer. Math. Soc. 349 (1997), no. 10, 4251-4264. MR 97m:20049

[21] G. D. Mostow, Strong rigidity of locally symmetric spaces, Annals of Mathematics Studies, No. 78. Princeton University Press, Princeton, N.J.; University of Tokyo Press, Tokyo, 1973. MR 52:5874

[22] Otal, J-P, Le théorème d'hyperbolisation pour les variétés fibrées de dimension 3, Astérisque No. 235 (1996). MR 97e:57013

[23] J. Ratcliffe, Foundations of hyperbolic manifolds, Graduate Texts in Mathematics, 149. Springer-Verlag, New York, 1994. MR 95j:57011

[24] G. P. Scott, Finitely generated 3-manifold groups are finitely presented, J. London Math. Soc. (2) 6 (1973), 437-440. MR 52:1660

[25] G. P. Scott, Compact submanifolds of 3-manifolds, J. London Math. Soc. 7 (1973), 246-250. MR 48:5080

[26] P. Scott, Normal subgroups in 3-manifold groups, J. London Math. Soc. (2) 13 (1976), no. 1, 5-12. MR 53:6565

[27] P. Scott, A new proof of the annulus and torus theorems, Amer. J. Math. 102 (1980), no. 2, 241-277. MR 81f:57006

[28] P. Shalen, Infinitely divisible elements in 3-manifold groups, Knots, groups, and 3-manifolds (Papers dedicated to the memory of R. H. Fox), pp. 293-335. Ann. of Math. Studies, No. 84, Princeton Univ. Press, Princeton, N.J., 1975. MR 51:11476

[29] J. Simon, Compactifications of covering spaces of compact 3-manifolds, Michigan Math. J. 23 (1976), 245-256. MR 55:4178

[30] J. Stallings, On fibering certain 3-manifolds, 1962 Topology of 3-manifolds and related topics (Proc. The Univ. of Georgia Institute, 1961) pp. 95-100 Prentice-Hall, Englewood Cliffs, N.J. MR 28:1600

[31] J. Stallings and S. Gersten, Casson's idea about 3-manifolds whose universal cover is $R^{3}$ Internat. J. Algebra Comput. 1 (1991), no. 4, 395-406. MR 93b:57018

[32] W. P. Thurston, Three-dimensional manifolds, Kleinian groups and hyperbolic geometry, Bull. Amer. Math. Soc. (N.S.) 6 (1982), no. 3, 357-381. MR 83h:57019

[33] T. Tucker, Non-compact 3-manifolds and the missing-boundary problem, Topology 13 (1974), 267-273. MR 50:5801

[34] F. Waldhausen, On irreducible 3-manifolds which are sufficiently large, Ann. of Math., 87 (1968), 56-88. MR 36:7146

Department of Mathematics, Oklahoma State University, Stillwater, Oklahoma 74078

E-mail address: myersr@math.okstate.edu 